\title{
Nongeometric states in a holographic conformal field theory
}

\author{
Wu-zhong Guo, ${ }^{1, *}$ Feng-Li Lin, ${ }^{2, \dagger}$ and Jiaju Zhang ${ }^{3,4, \hbar}$ \\ ${ }^{1}$ Physics Division, National Center for Theoretical Sciences, National Tsing Hua University, \\ No. 101, Sec. II, Kuang Fu Road, Hsinchu 30013, Taiwan \\ ${ }^{2}$ Department of Physics, National Taiwan Normal University, No. 88, Sec. IV, Ting-Chou Road, \\ Taipei 11677, Taiwan \\ ${ }^{3}$ Dipartimento di Fisica G. Occhialini, Università degli Studi di Milano-Bicocca, \\ Piazza della Scienza 3, 20126 Milano, Italy \\ ${ }^{4}$ INFN, Sezione di Milano-Bicocca, Piazza della Scienza 3, 20126 Milano, Italy
}

(Received 7 July 2018; published 7 May 2019)

\begin{abstract}
In the $\mathrm{AdS}_{3} / \mathrm{CFT}_{2}$ correspondence, we find some conformal field theory (CFT) states that have no bulk description by the Bañados geometry. We elaborate the constraints for a CFT state to be geometric, i.e., having a dual Bañados metric, by comparing the order of central charge of the entanglement/Rényi entropy obtained respectively from the holographic method and the replica trick in CFT. We find that the geometric CFT states fulfill Bohr's correspondence principle by reducing the quantum Korteweg-de Vries hierarchy to its classical counterpart. We call the CFT states that satisfy the geometric constraints geometric states, and otherwise, we call them nongeometric states. We give examples of both the geometric and nongeometric states, with the latter case including the superposition states and descendant states.
\end{abstract}

DOI: 10.1103/PhysRevD.99.106001

\section{INTRODUCTION}

The anti-de Sitter (AdS)/conformal field theory (CFT) correspondence conjectures that the bulk quantum gravity is equivalent to the boundary CFT [1]. In the semiclassical limit of bulk theory, a CFT state is believed to be dual to a bulk geometry if the quantum fluctuation can be minimized. We call such a kind of CFT states the geometric states. Thus, it is easy to see that the superposition of two geometric states cannot be geometric because the superposition principle should not hold for the bulk classical gravity [2]. Despite there being many discussions on the criterion for a CFT state to be geometric, e.g., Refs. [3,4] and a review in Ref. [5], it still lacks a concise criterion that one can adopt to check for more generic cases. For example, in $\mathrm{AdS}_{5} / \mathrm{CFT}_{4}$ correspondence, people know that the vacuum state of $\mathrm{SU}(N)$ gauge theory admits only planar correlators in the large $N$ limit, which is then dual to classical gravity in pure $\mathrm{AdS}_{5}$ space. In this case, the quantum fluctuation of nonplanar diagrams is suppressed, and a bulk geometry is emerging as the holographic dual.

\footnotetext{
*wzguo@cts.nthu.edu.tw

†linfengli@phy.ntnu.edu.tw

\#iaju.zhang@unimib.it
}

Published by the American Physical Society under the terms of the Creative Commons Attribution 4.0 International license. Further distribution of this work must maintain attribution to the author(s) and the published article's title, journal citation, and DOI. Funded by SCOAP ${ }^{3}$.
However, there is no clear planar limit for arbitrary excited states.

The situation becomes sharper in three-dimensional (3D) AdS gravity, which is dual to a two-dimensional (2D) CFT [6], and thus the bulk Bañados geometries [7] are determined by the expectation value of the stress tensor of dual 2D CFT states in the large central charge $c$ limit. Due to the topological nature of 3D AdS gravity, we can state that the 2D geometric states should be described by the Bañados geometries. The primary states and canonical ensemble states are known to be described by the Bañados geometries as can be verified by the match of entanglement entropy and its holographic dual [8,9] in the Bañados-TeitelboimZanelli black hole [10] background. Here, $c$ plays a similar role as $N$ in the $\mathrm{AdS}_{5} / \mathrm{CFT}_{4}$; however, there is no analog of the planar limit even for the vacuum state to define the suppression of quantum fluctuation. Naively, one can require the standard deviation/uncertainty of any local operator to be small as the criterion for the suppression of quantum fluctuation, and thus the geometric states. However, the question is what the exact suppression order of these standard deviations/uncertainties should be in the large $c$ expansion. We need a concise criterion to check for more generic (non)geometric states, at least in $\mathrm{AdS}_{3} / \mathrm{CFT}_{2}$.

In this work, we formulate such a criterion by comparing the nonlocal observables such as entanglement entropy and Rényi entropy with their holographic duals $[8,9,11]$. If the CFT state is geometric, then its entanglement/Rényi entropy calculated á la the replica trick [12-15] should agree with the corresponding holographic dual calculated 
from the dual Bañados geometries. Otherwise, it is nongeometric. Moreover, by short-interval expansion, we can turn this criterion into the constraints on the standard deviation of the stress tensors and its higher order cousins in terms of Korteweg-de Vries (KdV) charges. This will then tell precisely how much the quantum fluctuation should be suppressed for a state to be geometric. With such a concrete criterion, we indeed find some new nongeometric states, which are descendant states.

Our paper is organized as follows. In Sec. II, we state explicitly our criterion for the geometric CFT states. In Sec. III, we derive the conditions for geometric CFT states on the expectation values of quasiprimaries. In Sec. IV, we demonstrate a correspondence principle for the $\mathrm{KdV}$ charges for the geometric CFT states. We then give the examples for the geometric CFT states and nongeometric CFT states in Secs. V and VI, respectively. Finally, we conclude our paper in Sec. VII with discussions on our geometric state conditions and the connected correlation functions characterizing the suppression of quantum fluctuations. Besides, we elaborate technical details in various Appendixes. In Appendix A, we give the more explicit details of the conditions given in Sec. III for geometric CFT states. In Appendix B, we elaborate the derivation of the conditions in Sec. III and Appendix A. In Appendix C, we give the detailed check for a coordinate-dependent example of the geometric state discussed in Sec. V. In Appendix D, we elaborate the check of nongeometric descendant states discussed in Sec. VI.

\section{CRITERION FOR GEOMETRIC CFT STATES IN BAÑADOS GEOMETRY}

Due to the topological nature of 3D Einstein gravity, i.e., that there is no bulk propagating degree of freedom, the bulk geometry is completely determined by the asymptotic boundary constraints; this led Bañados to conjecture that all the vacuum asymptotically $\mathrm{AdS}_{3}$ solutions of 3D Einstein gravity are completely classified by the boundary conformal symmetries. Applying this conjecture to AdS/CFT correspondence, it leads to the Bañados geometries, which are determined by the expectation value of the stress tensor with respect to the dual CFT state. More precisely, the form of the Bañados geometry takes the form [7]

$d s^{2}=\frac{d y^{2}}{y^{2}}+\frac{L_{\rho}}{2} d z^{2}+\frac{\bar{L}_{\rho}}{2} d \bar{z}^{2}+\left(\frac{1}{y^{2}}+\frac{y^{2}}{4} L_{\rho} \bar{L}_{\rho}\right) d z d \bar{z}$,

where we set the AdS radius to unity $R=1$ so that the bulk Newton constant $G_{N}$ is related to the central charge $c$ of the dual CFT by $c=\frac{3}{2 G_{N}}[6]$.

We consider a holographic CFT on a cylinder with complex coordinate $w$ and spatial period $L$ in a state with density matrix $\rho$, and the cylinder can be mapped to a complex plane with coordinate $z$ by the conformal transformation $z=\mathrm{e}^{\frac{2 \pi i v}{L}}$. The functions $L_{\rho}(z)$ and $\bar{L}_{\rho}(\bar{z})$ in the Bañados geometry are respectively holomorphic and antiholomorphic and are related to the expectation value of the stress tensor on the plane with respect to the dual CFT state

$$
\langle T(z)\rangle_{\rho}=-\frac{c}{12} L_{\rho}(z), \quad\langle\bar{T}(\bar{z})\rangle_{\rho}=-\frac{c}{12} \bar{L}_{\rho}(\bar{z}) .
$$

Given a Bañados geometry which is dual to a CFT state $\rho$, one can then evaluate the holographic entanglement/ Rényi entropy á la the prescriptions in Refs. [8,9,11]. Both the holographic entanglement and Rényi entropies are given by the area law formula. If we consider a CFT state, for which $\langle T(z)\rangle_{\rho}$ and $\langle\bar{T}(z)\rangle_{\rho}$ are of order $c$, then the metric of the dual Bañados geometry is of order $c^{0}$ in the large $c$ expansion and should be independent of $c$ in the large $c$ limit. Thereby, the area of minimal surface or cosmic brane should be independent of $c$ so that the holographic entanglement/Rényi entropies should be of order $c$ due to the relation $c=\frac{3}{2 G_{N}}$. Based on the above result, we now formulate our criterion for the geometric CFT states. For a 2D CFT state of order $c$ stress tensor expectation value to be holographic dual to a Bañados geometry, the entanglement/Rényi entropy obtained from CFT calculations should be at most order $c$ in the large $c$ limit. Otherwise, we call the CFT state nongeometric.

\section{CONSTRAINTS FOR GEOMETRIC CFT STATES}

Based on our proposed criterion for the geometric CFT states, i.e., that the entanglement/Rényi entropy should be at most of order $c$ in the large $c$ limit, we would like to extract the necessary constraints by explicitly evaluating the entanglement/Rènyi entropy. The prescription of evaluating entanglement/Rényi entropy is based on the replica trick [16], which leads to an $n$-fold CFT that we call CFT ${ }^{n}$. However, there is usually no closed form of entanglement/ Rényi entropy for generic excited states. Instead, we will evaluate in the short-interval expansion, similar to what has been done in Refs. [12-15]. By assuming dominance of the vacuum conformal family in the operator product expansion (OPE) of twist operators [17-20] in the large $c$ limit, the entanglement/Rényi entropy takes the formal form in terms of the series of expectation values of $\mathrm{CFT}^{n}$ quasiprimary fields $\Phi_{K}$ that are constructed by operators in the vacuum conformal family of the original one-fold CFT. Since the contributions from the holomorphic and antiholomorphic sectors decouple and are similar, in this paper, we only consider the contributions from the holomorphic sector.

We consider the short interval $A=[w, w+\ell]$ with $\ell \ll L$, and from the OPE of twist operators, we get the short-interval expansion of the Rényi entropy, 


$$
\begin{aligned}
S_{A, \rho}^{(n)}= & \frac{c(n+1)}{12 n} \log \frac{\ell}{\epsilon} \\
& -\frac{1}{n-1} \log \left(\sum_{K} d_{K} \sum_{r=0}^{\infty} \frac{a_{K}^{r}}{r !} \ell^{h_{K}+r}\left\langle\Phi_{K}^{(r)}(w)\right\rangle_{\rho}\right) .
\end{aligned}
$$

The summation of $K$ is over all the $\mathrm{CFT}^{n}$ holomorphic quasiprimary operators $\Phi_{K}$, with conformal weight $h_{K}$, which are constructed from the holomorphic quasiprimary operators in the original one-fold CFT. The forms of $\Phi_{K}$ to level 8, which are constructed from $T$ at level $2 ; \mathcal{A}$ at level $4 ; \mathcal{B}$ and $\mathcal{D}$ at level 6 ; and $\mathcal{E}, \mathcal{H}$, and $\mathcal{I}$ at level 8 as well as their corresponding OPE coefficients $d_{K}$, can be found in Ref. [21]. There is the coefficient $a_{K}^{r}=C_{h_{K}+r-1}^{r} / C_{2 h_{K}+r-1}^{r}$.

Requiring that the Rényi entropy of $A$ in state $\rho$ is of at most order $c$, we get the constraints for the one-point functions up to level 6 ,

$$
\begin{aligned}
& \langle T\rangle_{\rho}=c \alpha(w)+\beta(w)+\frac{\gamma(w)}{c}+O\left(\frac{1}{c^{2}}\right), \\
& \langle\mathcal{A}\rangle_{\rho}=c^{2} \alpha(w)^{2}+c \delta(w)+\epsilon(w)+O\left(\frac{1}{c}\right), \\
& \langle\mathcal{B}\rangle_{\rho}=c^{2}\left[\alpha^{\prime}(w)^{2}-\frac{4}{5} \alpha(w) \alpha^{\prime \prime}(w)\right]+O(c), \\
& \langle\mathcal{D}\rangle_{\rho}=c^{3} \alpha(w)^{3}+3 c^{2} \alpha(w)[\delta(w)-\alpha(w) \beta(w)]+O(c),
\end{aligned}
$$

with $\alpha(w), \beta(w), \gamma(w), \delta(w)$, and $\epsilon(w)$ being arbitrary order $O\left(c^{0}\right)$ holomorphic functions.

We write the conditions (4) as the suggestive forms

$$
\begin{gathered}
\lim _{c \rightarrow \infty} \frac{\langle\mathcal{A}\rangle_{\rho}-\langle T\rangle_{\rho}^{2}}{c^{2}}=0, \\
\lim _{c \rightarrow \infty} \frac{\langle\mathcal{D}\rangle_{\rho}-3\langle\mathcal{A}\rangle_{\rho}\langle T\rangle_{\rho}+2\langle T\rangle_{\rho}^{3}}{c^{2}}=0 .
\end{gathered}
$$

Recall that $\mathcal{A}=(T T)-\frac{3}{10} \partial^{2} T$ with $(\cdots)$ denoting the normal ordering; $\sqrt{\langle\mathcal{A}\rangle_{\rho}-\langle T\rangle_{\rho}^{2}}$ plays the role of standard deviation of $T$ with respect to the geometric state $\rho$, and thus (5) tells that it should be smaller than order $c$ in the large $c$ limit. Similarly, $\mathcal{D}=(T(T T))+O\left(T^{2}\right)$; thus, Eq. (6) suggests that the uncertainty of cubic quantum fluctuation of $T$ should be also not larger than order $c$. There are more constraints at higher orders of $\ell$. See Appendixes A and B for more details.

Note that these constraints are in analogy to the planar limit of the large $N$ expansion in four-dimensional YangMills theory for the vacuum state. However, we are considering the excited state of large $c$ 2D CFTs, and there is no known planar limit for this case. Instead, our simple criterion serves as a guide for the analogy quantum suppression and yields the precise constraints for the geometric states. Next, we will justify the semiclassical nature of the geometric states for the physical observables in the sense of Bohr's correspondence principle.

\section{QUANTUM TO CLASSICAL KDV EQUATION AND CHARGES FOR GEOMETRIC CFT STATES}

The geometric state constraints relate the expectation values of operators in the vacuum family quasiprimaries. We will show that these constraints in fact reduce the quantum $\mathrm{KdV}$ equation and charges to their classical counterparts.

For demonstration, we write down the quantum $\mathrm{KdV}$ currents up to level 6 [22-24],

$$
J_{2}=T, \quad J_{4}=(T T), \quad J_{6}=(T(T T))-\frac{c+2}{12}\left(T^{\prime} T^{\prime}\right),
$$

with the parentheses denoting the normal ordering operators. In terms of the quasiprimary operators and their derivatives, we obtain

$$
\begin{aligned}
J_{2}= & T, \quad J_{4}=\mathcal{A}+\frac{3}{10} T^{\prime \prime}, \\
J_{6}= & \mathcal{D}-\frac{25(2 c+7)(7 c+68)}{108(70 c+29)} \mathcal{B} \\
& -\frac{2 c-23}{108} \mathcal{A}^{\prime \prime}-\frac{c-14}{280} T^{(4)} .
\end{aligned}
$$

These currents form the mutually commuting KdV charges

$$
Q_{2 k-1}=\int_{0}^{L} \frac{d w}{L} J_{2 k}(w),
$$

which constitute the integrability hierarchy of the quantum $\mathrm{KdV}$ equation

$$
\dot{T}=\frac{1-c}{6} T^{\prime \prime \prime}-3(T T)^{\prime}=-\frac{5 c+22}{30} T^{\prime \prime \prime}-3 \mathcal{A}^{\prime} .
$$

Using the leading order geometric state constraints (4), we set $\alpha(w)=U(w) / 6$ and get the classical $\mathrm{KdV}$ equation

$$
\dot{U}=U^{\prime \prime \prime}+6 U U^{\prime} .
$$

Note that $\partial_{t}$, which we denote by a dot, has been rescaled from the quantum $\mathrm{KdV}$ equation to its classical counterpart.

In the large $c$ limit, a natural definition of the classical counterpart of quantum KdV currents with respect to state $\rho$ is

$$
J_{2 k}^{\rho}(w) \equiv \lim _{c \rightarrow \infty} \frac{6^{k}}{c^{k}}\left\langle J_{2 k}(w)\right\rangle_{\rho} .
$$


Using the leading order of (4), we can then turn $J_{2 k}^{\rho}$ into the standard classical form

$$
J_{2}^{\rho}=U, \quad J_{4}^{\rho}=U^{2}, \quad J_{6}^{\rho}=U^{3}-\frac{1}{2} U^{\prime 2} .
$$

Their associated $\mathrm{KdV}$ charges constitute the integrability hierarchy of the classical KdV equation (11). This reflects Bohr's correspondence principle for these geometric states by reducing these $\mathrm{KdV}$ conserved currents into their classical counterparts.

In the textbook [25], the quantum to classical reduction for the $\mathrm{KdV}$ equation is obtained by simply replacing the $\mathrm{KdV}$ current operator with its classical counterpart without referring to the associated state. This does not work if the associated CFT state is nongeometric, as we discuss in this paper.

\section{EXAMPLES OF GEOMETRIC CFT STATES}

In Refs. [26-29], it has been shown that the Rényi entropy in the primary excited state

$$
\rho_{\phi}=\frac{1}{\alpha_{\phi}}|\phi\rangle\langle\phi|
$$

is of order $c$ if the conformal weight $h_{\phi}$ is at most of order $c$, so the expectation values of quasiprimaries should satisfy all the geometric state constraints (4). This is also consistent with the calculation $[13,14]$ from the OPE of twist operators to order $\ell^{8}$.

Even without an explicit check as is done for the primary states, we can argue that some particular states should satisfy the geometric state constraints. For example, the thermal states which are dual to Bañados-TeitelboimZanelli black holes thus should also be geometric. Similarly, the states which are conformally related to the vacuum state on the plane, denoted by $|0\rangle$, should also be geometric. In the bulk, these states are dual to the Bañados geometries, which can be transformed to pure $\mathrm{AdS}_{3}$ by the coordinate transformation dual to the boundary conformal map. These states include the thermal state and the conical defect state.

In quantum mechanics, the wave packet state behaves like a classical particle. This motivates us now to check if a wave packet state can also have the bulk description. Explicitly, the state considered has the density matrix

$$
\begin{aligned}
\rho_{\phi\left(w_{0}\right)} & =\frac{1}{\alpha_{\phi}}\left[\frac{L}{\pi} \sin \frac{\pi\left(\bar{w}_{0}-w_{0}\right)}{L}\right]^{2 h_{\phi}} \phi\left(w_{0}\right)|0\rangle\langle 0| \phi\left(\bar{w}_{0}\right) \\
& =\frac{1}{\alpha_{\phi}}\left(\frac{1-z_{0} \bar{z}_{0}}{\bar{z}_{0}}\right)^{2 h_{\phi}} \phi\left(z_{0}\right)|0\rangle\langle 0| \phi\left(1 / \bar{z}_{0}\right) .
\end{aligned}
$$

Note that $w_{0}$ is a position on the cylinder and $z_{0}$ is a position on the plane with the relation $z_{0}=\mathrm{e}^{\frac{2 \pi i w_{0}}{L}}$. Since $\phi\left(z_{0}\right)|0\rangle=\mathrm{e}^{z_{0} L_{-1}}|\phi\rangle$, the above state can be understood as a coherent sum of the primary state $|\phi\rangle$ and its global descendants. We check that the one-point functions in the state $\rho_{\phi\left(w_{0}\right)}$ satisfy the constraints (4). See Appendix C for details. This is consistent with the fact that on the cylinder the locally excited state is dual to a moving particle in $\mathrm{AdS}_{3}$ [26,30], i.e., that there exists a bulk geometric description.

\section{EXAMPLES OF NONGEOMETRIC CFT STATES}

From our discussions, we see that there is an infinite tower of constraints for a state to be geometric. Then, it seems that it should be quite easy to have nongeometric states by violating one of the infinite number of constraints. The reason why we did not know any example of nongeometric states is partly due to lack of principle of check as proposed in this work and partly due to the technical involvement of evaluating the geometric state constraints. In the following, we will consider some examples of nongeometric states, for which we know how to evaluate the associated one-point functions of the vacuum family quasiprimary operators to check (4).

As discussed in the Introduction, one expects the superposition of primary states will not be geometric because the bulk gravity is classical, so the superposition principle does not work. Now, we would like to check this explicitly.

Let us choose $\left|\phi_{1}\right\rangle$ and $\left|\phi_{2}\right\rangle$ as two primary states with conformal weights $h_{\phi_{1}}=c \epsilon_{\phi_{1}}+O\left(c^{0}\right), h_{\phi_{2}}=c \epsilon_{\phi_{2}}+O\left(c^{0}\right)$, and $\epsilon_{\phi_{1}} \neq \epsilon_{\phi_{2}}$. We consider the superposition state

$$
\cos (\theta)\left|\phi_{1}\right\rangle+\mathrm{e}^{\mathrm{i} \psi} \sin (\theta)\left|\phi_{2}\right\rangle
$$

The constraints (4) are satisfied separately for the states $\left|\phi_{1}\right\rangle$ and $\left|\phi_{2}\right\rangle$; however, they are violated for the superposition state (16). This means that the superposition of two primary states is nongeometric, as we expect. It is straightforward to generalize the above result to superposition states $\sum_{i} c_{i}\left|\phi_{i}\right\rangle$ with $\left|\phi_{i}\right\rangle$ 's being different primary states.

Other examples that do not satisfy the constraints (4) are some descendant states

$$
\begin{aligned}
& \left|\phi^{(m)}\right\rangle \quad \text { with } \quad h_{\phi}+m \sim O(c), \\
& |\tilde{\phi}\rangle \quad \text { with } \quad h_{\phi} \sim O(c), \\
& \left|\tilde{\phi}^{(m)}\right\rangle \quad \text { with } \quad h_{\phi}+m \sim O(c), \\
& \left|T^{(m)}\right\rangle \quad \text { with } \quad m \sim O(c), \\
& \left|\mathcal{A}^{(m)}\right\rangle \quad \text { with } \quad m \sim O(c),
\end{aligned}
$$

where $\phi$ is a primary operator and $\tilde{\phi}$ is a quasiprimary operator with the definition $\tilde{\phi} \equiv(T \phi)-\frac{3}{2\left(h_{\phi}+1\right)} \phi^{\prime \prime}$. Note that we have not yet normalized these descendant states properly. By $h_{\phi}+m \sim O(c)$, we mean that either $h_{\phi}$ or $m$ can be of order $O\left(c^{0}\right)$ or $O(c)$, but the sum $h_{\phi}+m$ is of order $O(c)$. See more details in Appendix D. 
Among the examples of nongeometric states, the superposition states can be understood intuitively. On the other hand, we have no immediate understanding as to why the descendant states lack the bulk classical geometric descriptions. In Ref. [31], the descendant states are understood as the dressings of gravitons on the particle's worldline. It is hard to see why some of the dressings cannot be backreacted geometrically, especially for the case with $m$ being $O\left(c^{0}\right)$. We may then ask if these states will turn to be geometric if quantum gravity effects are taken into account. In the context of perturbative quantum gravity by including higher derivative curvature terms, the answer is no because these terms are of higher orders in $G_{N} \sim 1 / c$, so they can only yield subleading order $1 / c$ corrections to the Bañados geometry, and the holographic entanglement/Rényi entropies remain order $c$. Therefore, we are forced to accept the existence of these nongeometric states, or the quantum gravity correction should be nonperturbative.

Moreover, in the context of quantum thermalization and canonical typicality $[32,33]$ the nongeometric states are obviously the atypical states because their entanglement/ Rényi entropies are quite different from the ones of thermal states. Using the result in Ref. [34], it can be shown that there are more descendant states than the primary ones at high levels in the large $c$ limit [35]. If most of these descendant states are nongeometric, one would then expect the canonical typicality to fail for 2D large $c$ CFTs.

\section{CONCLUSIONS}

In this work, based on (holographic) entanglement entropy, we have formulated a criterion to check if a 2D CFT state can have a bulk geometric description or not. Moreover, we derive the explicit constraints for an explicit check and find that all the primary states are geometric along with the discovery of some nongeometric states.

In this concluding section, we elaborate the relation between our geometric state conditions and the connected correlation functions, which characterize the suppression of the quantum fluctuations.

In statistical mechanics, the connected correlation function or Ursell function of multivariate random variables is defined by

$U_{n}\left(X_{1}, X_{2}, \ldots, X_{n}\right):=\left.\frac{\partial}{\partial \xi_{1}} \frac{\partial}{\partial \xi_{2}} \ldots \frac{\partial}{\partial \xi_{n}} \log \left\langle e^{\sum_{i} \xi_{i} X_{i}}\right\rangle\right|_{\xi_{i}=0}$,

where $\langle\cdots\rangle$ means taking the expectation value of the variables. For our purpose, we take $X_{i}$ to be the stress energy tensor $T\left(z_{i}\right)$ at point $z_{i}$ and the expectation value to be $\langle\ldots\rangle_{\rho}$. We denote the connected correlation functions of $T$ by $U_{n}^{\rho}\left(T\left(z_{1}\right), T\left(z_{2}\right), \ldots, T\left(z_{n}\right)\right)$, and the first few of them are given by

$$
\begin{aligned}
U_{1}^{\rho}\left(T\left(z_{1}\right)\right)= & \left\langle T\left(z_{1}\right)\right\rangle \\
U_{2}^{\rho}\left(T\left(z_{1}\right), T\left(z_{2}\right)\right)= & \left\langle T\left(z_{1}\right) T\left(z_{2}\right)\right\rangle_{\rho}-\left\langle T\left(z_{1}\right)\right\rangle_{\rho}\left\langle T\left(z_{2}\right)\right\rangle, \\
U_{3}^{\rho}\left(T\left(z_{1}\right), T\left(z_{2}\right), T\left(z_{3}\right)\right)= & \left\langle T\left(z_{1}\right) T\left(z_{2}\right) T\left(z_{3}\right)\right\rangle_{\rho}-\left\langle T\left(z_{1}\right)\right\rangle_{\rho}\left\langle T\left(z_{2}\right) T\left(z_{3}\right)\right\rangle_{\rho}-\left\langle T\left(z_{2}\right)\right\rangle_{\rho}\left\langle T\left(z_{1}\right) T\left(z_{3}\right)\right\rangle_{\rho} \\
& -\left\langle T\left(z_{3}\right)\right\rangle_{\rho}\left\langle T\left(z_{1}\right) T\left(z_{2}\right)\right\rangle_{\rho}+2\left\langle T\left(z_{1}\right)\right\rangle_{\rho}\left\langle T\left(z_{2}\right)\right\rangle_{\rho}\left\langle T\left(z_{3}\right)\right\rangle_{\rho} .
\end{aligned}
$$

We could also generalize to operator $\partial^{m} T$, for examples,

$$
\begin{aligned}
U_{2}^{\rho}\left(\partial T\left(z_{1}\right), \partial T\left(z_{2}\right)\right)= & \left\langle\partial T\left(z_{1}\right) \partial T\left(z_{2}\right)\right\rangle_{\rho} \\
& -\left\langle\partial T\left(z_{1}\right)\right\rangle_{\rho}\left\langle\partial T\left(z_{2}\right)\right\rangle_{\rho} \\
U_{2}^{\rho}\left(\partial^{2} T\left(z_{1}\right), T\left(z_{2}\right)\right)= & \left\langle\partial^{2} T\left(z_{1}\right) T\left(z_{2}\right)\right\rangle_{\rho} \\
& -\left\langle\partial^{2} T\left(z_{1}\right)\right\rangle_{\rho}\left\langle T\left(z_{2}\right)\right\rangle_{\rho} .
\end{aligned}
$$

We derive the geometric conditions on the cylinder with coordinate $w$ and spatial period $L$, but now it is convenient to work on the complex plane with coordinate $z$. We would like to show that the geometric conditions are invariant under a conformal map $z=f(w)$. After performing a conformal map $z=f(w)$ on the relation $S_{A, \rho}^{(n)}(w) \sim$ $\log \left\langle\sigma_{n}\left(w_{1}\right) \tilde{\sigma}_{n}\left(w_{2}\right)\right\rangle_{\rho^{n}}$, we then have

$S_{A, \rho}^{(n)}(z) \sim \log \left\langle\sigma_{n}\left(z_{1}\right) \tilde{\sigma}_{n}\left(z_{2}\right)\right\rangle_{\rho^{n}}+h_{n} \log \left(f^{\prime}\left(w_{1}\right) f^{\prime}\left(w_{2}\right)\right)$, where $h_{n}=\frac{c}{24}(n-1 / n)$. Therefore, the requirement $S_{A, \rho}^{(n)}(w) \sim O(c)$ is equivalent to $S_{A, \rho}^{(n)}(z) \sim O(c)$. We further use the OPE of twist operators on the plane with the coordinate $z$ and get exactly the same conditions for the one-point functions of quasiprimary operators with $\Phi_{K}(w)$ replaced by $\Phi_{K}(z)$. By a conformal map $z=e^{2 \pi i w / L}$, the cylinder is mapped to the complex plane. If the conditions on the plane are justified, it leads to the justification of the conditions on the cylinder.

On the complex plane, one may rewrite the first geometric state condition (B5) as

$$
\frac{1}{2 \pi i} \oint_{z_{2}} \frac{d z_{1}}{z_{1}-z_{2}}\left(\lim _{c \rightarrow \infty} \frac{U_{2}^{\rho}\left(T\left(z_{1}\right), T\left(z_{2}\right)\right)}{c^{2}}\right)=0 .
$$

Similarly, for the condition (B11), we have 


$$
\begin{aligned}
\langle\mathcal{D}\rangle_{\rho}-3\langle\mathcal{A}\rangle_{\rho}\langle T\rangle_{\rho}+2\langle T\rangle_{\rho}^{3} \\
=\langle(T(T T))\rangle_{\rho}-3\langle(T T)\rangle_{\rho}\langle T\rangle_{\rho} \\
\quad+2\langle T\rangle_{\rho}^{3}+\frac{9}{10}\left(\left\langle\left(\partial^{2} T T\right)\right\rangle_{\rho}\right. \\
\left.\quad-\left\langle\partial^{2} T\right\rangle_{\rho}\langle T\rangle_{\rho}\right)+O(c)
\end{aligned}
$$

and the condition

$$
\begin{aligned}
& \frac{1}{2 \pi i} \oint_{z_{3}} \frac{d z_{1}}{z_{1}-z_{3}} \frac{1}{2 \pi i} \oint_{z_{3}} \frac{d z_{2}}{z_{2}-z_{3}} \lim _{c \rightarrow \infty} \frac{1}{c^{2}} \\
& \quad \times\left[U_{3}^{\rho}\left(T\left(z_{1}\right), T\left(z_{2}\right), T\left(z_{3}\right)\right)+\frac{9}{10} U_{2}^{\rho}\left(\partial^{2} T\left(z_{2}\right), T\left(z_{3}\right)\right)\right] \\
& =0 .
\end{aligned}
$$

For the condition (B10), it is given by

$$
\begin{gathered}
\frac{1}{2 \pi i} \oint_{z_{2}} \frac{d z_{1}}{z_{1}-z_{2}} \lim _{c \rightarrow \infty} \frac{1}{c^{2}}\left[U_{2}^{\rho}\left(\partial T\left(z_{1}\right), \partial T\left(z_{2}\right)\right)\right. \\
\left.-\frac{4}{5} U_{2}^{\rho}\left(\partial^{2} T\left(z_{1}\right), T\left(z_{2}\right)\right)\right]=0 .
\end{gathered}
$$

Higher order conditions (B12), (B13), and (B14) can also be rewritten as the connected correlation functions. We will not show them here.

The geometric state conditions are in analogy to the planar limit of the correlation function of large $N$ expansion in four-dimensional Yang-Mills theory in a vacuum state. However, there is no solid argument to justify this analog. If one requires a stronger condition than the connected correlation functions of the scaled operator $T / c$,

$$
U_{n}^{\rho}\left(\partial^{m_{1}} T\left(z_{1}\right) / c, \partial^{m_{2}} T\left(z_{2}\right) / c, \ldots, \partial^{m_{n}} T\left(z_{n}\right) / c\right) \sim O\left(1 / c^{n-1}\right),
$$

for any integer $n$ and $m_{1}, \ldots, m_{n}$. It is just

$$
U_{n}^{\rho}\left(\partial^{m_{1}} T\left(z_{1}\right), \partial^{m_{2}} T\left(z_{2}\right), \ldots, \partial^{m_{n}} T\left(z_{n}\right)\right) \sim O(c) .
$$

The conditions (22), (24), and (25) will be satisfied. One could check the higher order conditions; they all should be satisfied. Note that the conditions we find are a criterion for generic excited states, not just for vacuum. We have checked that (27) is right for a primary state and thermal state up to $n=3$. It begs a quantum gravity interpretation of these conditions.

\section{ACKNOWLEDGMENTS}

We thank Bin Chen, Chong-Sun Chu, Bei-Lok Hu, Wei $\mathrm{Li}$, Wei Song, Chushun Tian, and Yong-Shi Wu for helpful discussions. W. Z. G. is supported by the National Center of Theoretical Science. F. L. L. is supported by Taiwan Ministry of Science and Technology through Grant
No. 106-2112-M-003-004-MY3. J.Z. is supported in part by Italian Ministero dell'Istruzione, Università e Ricerca, and Istituto Nazionale di Fisica Nucleare through the "Gauge Theories, Strings, Supergravity" research and by Fondazione Cariplo and Regione Lombardia, Grant No. 2015-1253.

\section{APPENDIX A: CONDITIONS FOR GEOMETRIC CFT STATES}

The conditions for geometric CFT states are expressed as one-point functions of quasiprimary operators in the vacuum family. We would like to summarize the definitions of these quasiprimary operators up to level 8; more details can be found in Refs. [15,20]. At level 2, we have the quasiprimary operator $T$. At level 4 , we have

$$
\mathcal{A}=(T T)-\frac{3}{10} \partial^{2} T \text {. }
$$

We use $(\mathcal{X Y})$ to denote normal ordering of $\mathcal{X}$ and $\mathcal{Y}$, and on the complex plane, it is defined as

$$
(\mathcal{X Y})(z)=\frac{1}{2 \pi i} \oint_{z} \frac{d w}{w-z} \mathcal{X}(w) \mathcal{Y}(z) .
$$

At level 6, we have two quasiprimary operators:

$$
\begin{aligned}
& \mathcal{B}=(\partial T \partial T)-\frac{4}{5}\left(\partial^{2} T T\right)-\frac{1}{42} \partial^{4} T, \\
& \mathcal{D}=(T(T T))-\frac{9}{10}\left(\partial^{2} T T\right)-\frac{1}{28} \partial^{4} T+\frac{93}{70 c+29} \mathcal{B} .
\end{aligned}
$$

At level 8, we have three quasiprimary operators,

$$
\begin{aligned}
\mathcal{E}= & \left(\partial^{2} T \partial^{2} T\right)-\frac{10}{9}\left(\partial^{3} T \partial T\right)+\frac{10}{63}\left(\partial^{4} T T\right)-\frac{1}{324} \partial^{6} T, \\
\mathcal{H}= & (\partial T(\partial T T))-\frac{4}{5}\left(\partial^{2} T(T T)\right)+\frac{2}{15}\left(\partial^{3} T \partial T\right)-\frac{3}{70}\left(\partial^{4} T T\right) \\
& +\frac{9(140 c+83)}{50(105 c+11)} \mathcal{E}, \\
\mathcal{I}= & (T(T(T T)))-\frac{9}{5}\left(\partial^{2} T(T T)\right)+\frac{3}{10}\left(\partial^{3} T \partial T\right) \\
& +\frac{81(35 c-51)}{100(105 c+11)} \mathcal{E}+\frac{12(465 c-127)}{5 c(210 c+661)-251} \mathcal{H} .
\end{aligned}
$$

\section{APPENDIX B: DERIVATION OF GEOMETRIC CONDITIONS}

By requiring the Rényi entropy [Eq. (3) in the main text] to be $O(c)$, we may get the following conditions, i.e., the conditions for geometric states. With some calculations, we get Rényi entropy up to $O\left(\ell^{8}\right)$, 


$$
\begin{aligned}
S_{A, \rho}^{(n)}= & \frac{c(n+1)}{12 n} \log \frac{\ell}{\epsilon}+\frac{\mathcal{C}_{2}}{1-n} \ell^{2}+\frac{\mathcal{C}_{3}}{1-n} \ell^{3}+\frac{2 \mathcal{C}_{4}-\mathcal{C}_{2}^{2}}{2(1-n)} \ell^{4}+\frac{\mathcal{C}_{5}-\mathcal{C}_{2} \mathcal{C}_{3}}{1-n} \ell^{5}+\frac{2 \mathcal{C}_{2}^{3}-3 \mathcal{C}_{3}^{2}-6 \mathcal{C}_{2} \mathcal{C}_{4}+6 \mathcal{C}_{6}}{6(1-n)} \ell^{6} \\
& +\frac{\mathcal{C}_{7}+\mathcal{C}_{2}^{2} \mathcal{C}_{3}-\mathcal{C}_{3} \mathcal{C}_{4}-\mathcal{C}_{2} \mathcal{C}_{5}}{1-n} \ell^{7}+\frac{4 \mathcal{C}_{8}-4 \mathcal{C}_{2} \mathcal{C}_{6}-4 \mathcal{C}_{3} \mathcal{C}_{5}-2 \mathcal{C}_{4}^{2}+4 \mathcal{C}_{2}^{2} \mathcal{C}_{4}+4 \mathcal{C}_{2} \mathcal{C}_{3} 2-\mathcal{C}_{2}^{4}}{4(1-n)} \ell^{8}+O\left(\ell^{9}\right)
\end{aligned}
$$

with

$$
\begin{aligned}
\mathcal{C}_{2}= & b_{T}\langle T\rangle_{\rho}, \quad \mathcal{C}_{3}=\frac{b_{T}}{2} \partial\langle T\rangle_{\rho}, \\
\mathcal{C}_{4}= & b_{\mathcal{A}}\langle\mathcal{A}\rangle_{\rho}+b_{T T}\langle T\rangle_{\rho}^{2}+\frac{3}{20} b_{T} \partial^{2}\langle T\rangle_{\rho} \\
\mathcal{C}_{5}= & \frac{1}{30} b_{T} \partial^{3}\langle T\rangle_{\rho}+\frac{1}{2} b_{\mathcal{A}} \partial\langle\mathcal{A}\rangle_{\rho}+\frac{1}{2} b_{T T} \partial\langle T\rangle_{\rho}^{2}, \\
\mathcal{C}_{6}= & b_{\mathcal{B}}\langle\mathcal{B}\rangle_{\rho}+b_{\mathcal{D}}\langle\mathcal{D}\rangle_{\rho}+b_{T \mathcal{A}}\langle T\rangle_{\rho}\langle\mathcal{A}\rangle_{\rho}+b_{T T T}\langle T\rangle_{\rho}^{3}+b_{\mathcal{K}} \mathcal{K}_{\rho}+\frac{1}{168} b_{T} \partial^{4}\langle T\rangle_{\rho}+\frac{5}{36} b_{\mathcal{A}} \partial^{2}\langle\mathcal{A}\rangle_{\phi}+\frac{5}{36} b_{T T} \partial^{2}\langle T\rangle_{\rho}^{2}, \\
\mathcal{C}_{7}= & \frac{1}{1120} b_{T} \partial^{5}\langle T\rangle_{\rho}+\frac{1}{36}\left[b_{\mathcal{A}} \partial^{3}\langle\mathcal{A}\rangle_{\rho}+b_{T T} \partial^{3}\left(\langle T\rangle_{\rho}^{2}\right)\right] \\
& +\frac{1}{2}\left[b_{\mathcal{B}} \partial\langle\mathcal{B}\rangle_{\rho}+b_{\mathcal{D}} \partial\langle\mathcal{D}\rangle_{\rho}+b_{T \mathcal{A}} \partial\left(\langle T\rangle_{\rho}\langle\mathcal{A}\rangle_{\rho}\right)+b_{T T T} \partial\left(\langle T\rangle_{\rho}^{3}\right)+b_{\mathcal{K}} \partial \mathcal{K}_{\rho}\right], \\
\mathcal{C}_{8}= & b_{\mathcal{E}}\langle\mathcal{E}\rangle_{\rho}+b_{\mathcal{H}}\langle\mathcal{H}\rangle_{\rho}+b_{\mathcal{I}}\langle\mathcal{I}\rangle_{\rho}+b_{T \mathcal{B}}\langle T\rangle_{\rho}\langle\mathcal{B}\rangle_{\rho}+b_{T \mathcal{D}}\langle T\rangle_{\rho}\langle\mathcal{D}\rangle_{\rho}+b_{\mathcal{A} \mathcal{A}}\langle\mathcal{A}\rangle_{\rho}^{2}+b_{T T \mathcal{A}}\langle T\rangle_{\rho}^{2}\langle\mathcal{A}\rangle_{\rho}+b_{T T T T}\langle T\rangle_{\rho}^{4} \\
& +b_{T \mathcal{K}}\langle T\rangle_{\rho} \mathcal{K}_{\rho}+b_{\mathcal{O}} \mathcal{O}_{\rho}+b_{\mathcal{P}} \mathcal{P}_{\rho}+b_{\mathcal{Q}} \mathcal{Q}_{\rho}+b_{\mathcal{R}} \mathcal{R}_{\rho}+\frac{1}{8640} b_{T} \partial^{6}\langle T\rangle_{\rho}+\frac{7}{1584} b_{\mathcal{A}} \partial^{4}\langle\mathcal{A}\rangle_{\rho}+\frac{7}{1584} b_{T T} \partial^{4}\langle T\rangle_{\rho}^{2} \\
& +\frac{7}{52}\left[b_{\mathcal{B}} \partial^{2}\langle\mathcal{B}\rangle_{\rho}+b_{\mathcal{D}} \partial^{2}\langle\mathcal{D}\rangle_{\rho}+b_{T \mathcal{A}} \partial^{2}\left(\langle T\rangle_{\rho}\langle\mathcal{A}\rangle_{\rho}\right)+b_{T T T} \partial^{2}\langle T\rangle_{\rho}^{3}+b_{\mathcal{K}} \partial^{2} \mathcal{K}_{\rho}\right] .
\end{aligned}
$$

The expectation values $\langle\mathcal{X}\rangle_{\rho}=\langle\mathcal{X}(w)\rangle_{\rho}, \mathcal{X}=T, \mathcal{A}, \mathcal{B}, \mathcal{D}, \cdots$, are functions of the coordinate $w$. The coefficients $b_{K}$ are defined in Ref. [20] from the OPE coefficients $d_{K}$ of the twist operators and are constants depending on $n$ and $c$. There are also definitions

$$
\begin{aligned}
\mathcal{K}_{\rho} & =\left(\partial\langle T\rangle_{\rho}\right)^{2}-\frac{4}{5}\langle T\rangle_{\rho} \partial^{2}\langle T\rangle_{\rho}, \\
\mathcal{O}_{\rho} & =\partial\langle T\rangle_{\rho} \partial\langle\mathcal{A}\rangle_{\rho}-\frac{2}{9}\langle T\rangle_{\rho} \partial^{2}\langle\mathcal{A}\rangle_{\rho}-\frac{4}{5} \partial^{2}\langle T\rangle_{\rho}\langle\mathcal{A}\rangle_{\rho}, \\
\mathcal{P}_{\rho} & =\left(\partial^{2}\langle T\rangle_{\rho}\right)^{2}-\frac{10}{9} \partial\langle T\rangle_{\rho} \partial^{3}\langle T\rangle_{\rho}+\frac{10}{63}\langle T\rangle_{\rho} \partial^{4}\langle T\rangle_{\rho}, \\
\mathcal{Q}_{\rho} & =\frac{7}{9}\langle T\rangle_{\rho} \mathcal{K}_{\rho}, \quad \mathcal{R}_{\rho}=\frac{7}{11}\langle T\rangle_{\rho} \mathcal{K}_{\rho} .
\end{aligned}
$$

At $O\left(\ell^{4}\right)$, we have

$$
2 \mathcal{C}_{4}-\mathcal{C}_{2}^{2}=\frac{n^{2}-1}{720 n^{3}}\left[\left(n^{2}-1\right)\left(\langle\mathcal{A}\rangle_{\rho}-\langle T\rangle_{\rho}^{2}\right)+18 n^{2} \partial^{2}\langle T\rangle_{\rho}\right]+O(1 / c)
$$

The last term is $O(c)$; we get the first condition,

$$
\lim _{c \rightarrow \infty} \frac{\langle\mathcal{A}\rangle_{\rho}-\langle T\rangle_{\rho}^{2}}{c^{2}}=0
$$

At $O\left(\ell^{5}\right)$, we have

$$
\mathcal{C}_{5}-\mathcal{C}_{2} \mathcal{C}_{3}=\frac{n^{2}-1}{2880 n^{3}}\left[5\left(n^{2}-1\right)\left(\partial\langle\mathcal{A}\rangle_{\rho}-2\langle T\rangle_{\rho} \partial\langle T\rangle_{\rho}\right)+8 n^{2} \partial^{3}\langle T\rangle_{\rho}\right]+O(1 / c)
$$


This leads to the condition,

$$
\lim _{c \rightarrow \infty} \frac{\partial\langle\mathcal{A}\rangle_{\rho}-2\langle T\rangle_{\rho} \partial\langle T\rangle_{\rho}}{c^{2}}=0
$$

This is nothing but the derivative of (B5). At $O\left(\ell^{6}\right)$, we have

$$
\begin{aligned}
& 2 \mathcal{C}_{2}^{3}-3 \mathcal{C}_{3}^{2}-6 \mathcal{C}_{2} \mathcal{C}_{4}+6 \mathcal{C}_{6} \\
& =\frac{n^{2}-1}{60480 n^{5}}\left\{35\left(\langle\mathcal{D}\rangle_{\rho}-3\langle\mathcal{A}\rangle_{\rho}\langle T\rangle_{\rho}+2\langle T\rangle_{\rho}^{3}\right)+35\left(\langle\mathcal{B}\rangle_{\rho}-\mathcal{K}_{\rho}-2\left(\langle\mathcal{D}\rangle_{\rho}-3\langle\mathcal{A}\rangle_{\rho}\langle T\rangle_{\rho}+2\langle T\rangle_{\rho}^{3}\right)\right.\right. \\
& \left.\quad-5\left[\partial^{2}\langle\mathcal{A}\rangle_{\rho}-2\left(\partial\langle T\rangle_{\rho}\right)^{2}-2\langle T\rangle_{\rho} \partial^{2}\langle T\rangle_{\rho}\right]\right) n^{2}+7\left(5 \mathcal{K}_{\rho}-5\left(\partial\langle T\rangle_{\rho}\right)^{2}+4 \partial\langle T\rangle_{\rho} \partial^{2}\langle T\rangle_{\rho}\right) n^{3} \\
& \quad+35\left[\left(\langle\mathcal{B}\rangle_{\rho}-\mathcal{K}_{\rho}\right)-\left(\langle\mathcal{D}\rangle_{\rho}-3\langle\mathcal{A}\rangle_{\rho}\langle T\rangle_{\rho}+2\langle T\rangle_{\rho}^{3}\right)-5\left(\partial^{2}\langle\mathcal{A}\rangle_{\rho}-2\left(\partial\langle T\rangle_{\rho}\right)^{2}-2\langle T\rangle_{\rho} \partial^{2}\langle T\rangle_{\rho}\right)-\frac{36}{7} \partial^{4}\langle T\rangle_{\rho}\right] n^{4} \\
& \left.\quad-7\left(5 \mathcal{K}_{\rho}-5\left(\partial\langle T\rangle_{\rho}\right)^{2}+4 \partial\langle T\rangle_{\rho} \partial^{2}\langle T\rangle_{\rho}\right) n^{5}\right\}+O(1 / c) .
\end{aligned}
$$

By using the constraint (B5), we obtain

$$
\lim _{c \rightarrow \infty} \frac{\partial^{2}\langle\mathcal{A}\rangle_{\rho}-2\left(\partial\langle T\rangle_{\rho}\right)^{2}-2\langle T\rangle_{\rho} \partial^{2}\langle T\rangle_{\rho}}{c^{2}}=0
$$

Therefore, we will have the following conditions at this order:

$$
\begin{array}{r}
\lim _{c \rightarrow \infty} \frac{\langle\mathcal{B}\rangle_{\rho}-\mathcal{K}_{\rho}}{c^{2}}=0, \\
\lim _{c \rightarrow \infty} \frac{\langle\mathcal{D}\rangle_{\rho}-3\langle\mathcal{A}\rangle_{\rho}\langle T\rangle_{\rho}+2\langle T\rangle_{\rho}^{3}}{c^{2}}=0 .
\end{array}
$$

The expression of $O\left(\ell^{8}\right)$ is too lengthy, so we just list the results at this order,

$$
\begin{gathered}
\lim _{c \rightarrow \infty} \frac{1}{c^{2}}\left[\langle\mathcal{I}\rangle_{\rho}-4\langle\mathcal{D}\rangle_{\rho}\langle T\rangle_{\rho}-3\langle\mathcal{A}\rangle_{\rho}^{2}+12\langle\mathcal{A}\rangle_{\rho}\langle T\rangle_{\rho}^{2}+6\langle T\rangle_{\rho}^{4}\right]=0 \\
\lim _{c \rightarrow \infty} \frac{1}{c^{2}}\left[45\langle\mathcal{H}\rangle_{\rho}-65\langle\mathcal{B}\rangle_{\rho}\langle T\rangle_{\rho}+10\langle T\rangle_{\rho} \partial^{2}\langle\mathcal{A}\rangle_{\rho} 36\langle\mathcal{A}\rangle_{\rho} \partial^{2}\langle T\rangle_{\rho}-72\langle T\rangle_{\rho}^{2} \partial^{2}\langle T\rangle_{\rho}\right. \\
\left.-45 \partial\langle\mathcal{A}\rangle_{\rho} \partial\langle T\rangle_{\rho}+90\langle T\rangle_{\rho}\left[\partial\langle T\rangle_{\rho}\right]^{2}\right]=0 \\
\lim _{c \rightarrow \infty} \frac{1}{c^{2}}\left[\langle\mathcal{E}\rangle_{\rho}-\left[\partial^{2}\langle T\rangle_{\rho}\right]^{2}-10 / 63\left(\langle T\rangle_{\rho} \partial^{4}\langle T\rangle_{\rho}-7 \partial^{3}\langle T\rangle_{\rho} \partial\langle T\rangle_{\rho}\right)\right]=0
\end{gathered}
$$

Without loss of generality, we assume the one-point functions $\langle\mathcal{X}\rangle_{\rho}$ have the following forms,

$$
\begin{aligned}
& \langle T(w)\rangle_{\rho}=\sum_{k=-1}^{+\infty} c^{-k} t_{k}(w), \quad\langle\mathcal{A}(w)\rangle_{\rho}=\sum_{k=-2}^{+\infty} c^{-k} a_{k}(w), \quad\langle\mathcal{B}(w)\rangle_{\rho}=\sum_{k=-2}^{+\infty} c^{-k} b_{k}(w), \quad\langle\mathcal{D}(w)\rangle_{\rho}=\sum_{k=-3}^{+\infty} c^{-k} d_{k}(w) \\
& \langle\mathcal{E}(w)\rangle_{\rho}=\sum_{k=-2}^{+\infty} c^{-k} e_{k}(w), \quad\langle\mathcal{H}(w)\rangle_{\rho}=\sum_{k=-3}^{+\infty} c^{-k} h_{k}(w), \quad\langle\mathcal{I}(w)\rangle_{\rho}=\sum_{k=-4}^{+\infty} c^{-k} i_{k}(w),
\end{aligned}
$$


where $t_{k}(w), a_{k}(w), b_{k}(w), d_{k}(w), e_{k}(w)$, and $i_{k}(w)$ are arbitrary functions of order $O\left(c^{0}\right)$. The above geometric conditions give some relations among one-point functions $\langle\mathcal{X}\rangle_{\rho}$. The result is

$$
\begin{aligned}
\langle T\rangle_{\rho}= & c \alpha(w)+\beta(w)+\frac{\gamma(w)}{c}+O\left(\frac{1}{c^{2}}\right), \\
\langle\mathcal{A}\rangle_{\rho}= & c^{2} \alpha(w)^{2}+c \delta(w)+\epsilon(w)+O\left(\frac{1}{c}\right), \\
\langle\mathcal{B}\rangle_{\rho}= & c^{2}\left[\alpha^{\prime}(w)^{2}-\frac{4}{5} \alpha(w) \alpha^{\prime \prime}(w)\right]+c \zeta(w)+O\left(c^{0}\right), \\
\langle\mathcal{D}\rangle_{\rho}= & c^{3} \alpha(w)^{3}+3 c^{2} \alpha(w)[\delta(w)-\alpha(w) \beta(w)]+c \eta(w)+O\left(c^{0}\right), \\
\langle\mathcal{E}\rangle_{\rho}= & c^{2}\left\{\alpha^{\prime \prime}(w)^{2}+\frac{10}{63}\left[\alpha(w) \alpha^{(4)}(w)-7 \alpha^{\prime}(w) \alpha^{(3)}(w)\right]\right\}+O(c), \\
\langle\mathcal{H}\rangle_{\rho}= & c^{3} \alpha(w)\left[\alpha^{\prime}(w)^{2}-\frac{4}{5} \alpha(w) \alpha^{\prime \prime}(w)\right]+c^{2}\left[-\alpha^{\prime}(w)^{2} \beta(w)-2 \alpha(w) \alpha^{\prime}(w) \beta^{\prime}(w)+\frac{4}{5} \alpha(w)^{2} \beta^{\prime \prime}(w)\right. \\
& \left.+\frac{8}{5} \alpha(w) \alpha^{\prime \prime}(w) \beta(w)+\alpha^{\prime}(w) \delta^{\prime}(w)-\frac{4}{5} \alpha^{\prime \prime}(w) \delta(w)-\frac{2}{9} \alpha(w) \delta^{\prime \prime}(w)+\frac{13}{9} \alpha(w) \zeta(w)\right]+O(c), \\
\langle\mathcal{I}\rangle_{\rho}= & c^{4} \alpha(w)^{4}+2 c^{3} \alpha(w)^{2}[3 \delta(w)-4 \alpha(w) \beta(w)]+c^{2}\left[12 \alpha(w)^{2} \beta(w)^{2}+4 \alpha(w)^{3} \gamma(w)\right. \\
& \left.-12 \alpha(w) \beta(w) \delta(w)+3 \delta(w)^{2}-6 \alpha(w)^{2} \epsilon(w)+4 \alpha(w) \eta(w)\right]+O(c),
\end{aligned}
$$

with $\alpha(w), \beta(w), \gamma(w), \delta(w), \epsilon(w), \zeta(w)$, and $\eta(w)$ being arbitrary order $O\left(c^{0}\right)$ holomorphic functions.

\section{APPENDIX C: COORDINATE-DEPENDENT EXAMPLE}

Let us consider a state constructed by superposition of the primary state and its global descendants (on the complex plane),

$$
|\Psi\rangle:=\mathcal{N} \sum c_{m}\left|\partial^{m} \phi\right\rangle,
$$

where $\mathcal{N}$ is the normalization constant. For $c_{m}=\frac{z^{m}}{m !}$, we could write $\left|\Psi_{c}\right\rangle$ as the "coherent" state, i.e.,

$$
\left|\Psi_{c}\right\rangle=\mathcal{N} e^{z L_{-1}}|\phi\rangle, \quad \text { with } \quad \mathcal{N}=(1-\bar{z} z)^{h},
$$

where $h_{\phi}$ is the conformal dimension of $\phi$. It is obvious $\left|\Psi_{c}\right\rangle$ is a local state $O(z)|0\rangle$. We are interested in the expectation value of $\Phi_{K}(z)$ in $\left|\Psi_{c}\right\rangle$. Generally, we have

$$
\left\langle\Psi_{c}\left|\Phi_{K}(x)\right| \Psi_{c}\right\rangle=\mathcal{N}^{2} \sum_{s, t} \bar{c}_{s} c_{t}\left\langle\partial^{s} \phi\left|\Phi_{K}(x)\right| \partial^{t} \phi\right\rangle
$$

For $s \geq t$, we have

$$
\begin{aligned}
\left\langle\partial^{s} \phi\left|\Phi_{K}(x)\right| \partial^{t} \phi\right\rangle= & x^{s-t-h_{\Phi_{K}} t ! s !} \sum_{m \geq s-t}^{s} C_{s-t+m+h_{\Phi_{K}}}^{s-t+m} \\
& \times C_{m+h_{\Phi_{K}}-1}^{m} C_{2 h_{\phi}-h_{\Phi_{K}}+s-m-1},
\end{aligned}
$$

while for $s<t$,

$$
\begin{aligned}
\left\langle\partial^{s} \phi\left|\Phi_{K}(x)\right| \partial^{t} \phi\right\rangle= & x^{s-t-h_{\Phi_{K}}} t ! s ! \sum_{m \geq t-s}^{t} C_{t-s+m+h_{\Phi_{K}}-1}^{t-s+m} \\
& \times C_{m+h_{\Phi_{K}}-1}^{m} C_{2 h_{\phi}-h_{\Phi_{K}}+t-m-1}^{t-m} .
\end{aligned}
$$

From (C4) and (C5) into (C3), we get a simple result

$$
\left\langle\Psi_{c}\left|\Phi_{K}(x)\right| \Psi_{c}\right\rangle=C_{\phi \phi \Phi_{K}}\left(\frac{z \bar{z}-1}{(x-z)(1-\bar{z} x)}\right)^{h_{\Phi_{K}}}
$$

Using (C3)-(C5), we could calculate any state like the form (C1) as long as we know the coefficients $c_{n}$. One could check that the one-point functions in the state $\left|\Psi_{c}\right\rangle$ do satisfy all the geometric conditions. For example, the condition (B13) is

$$
\begin{gathered}
45\langle\mathcal{H}\rangle_{\rho}-65\langle\mathcal{B}\rangle_{\rho}\langle T\rangle_{\rho}+10\langle T\rangle_{\rho} \partial^{2}\langle\mathcal{A}\rangle_{\rho}+36\langle\mathcal{A}\rangle_{\rho} \partial^{2}\langle T\rangle_{\rho} \\
-72\langle T\rangle_{\rho}^{2} \partial^{2}\langle T\rangle_{\rho}-45 \partial\langle\mathcal{A}\rangle_{\rho} \partial\langle T\rangle_{\rho}+90\langle T\rangle_{\rho}\left[\partial\langle T\rangle_{\rho}\right]^{2} \\
=\frac{18 c \epsilon_{\phi}\left(1845 c \epsilon_{\phi}-385 c+28\right)\left(z z^{*}-1\right)^{8}}{35(105 c+11)(x-z)^{8}\left(x z^{*}-1\right)^{8}} \sim O(c),
\end{gathered}
$$

where we define $\epsilon_{\phi}=h_{\phi} / c$. But if we slightly change the coefficients $c_{m}=\frac{z^{m}}{m !}$, it is very likely the corresponding state will violate the constraints. At least in this example, we can see the geometric conditions we find are highly nontrivial. 


\section{APPENDIX D: NONGEOMETRIC DESCENDANT STATES}

In the main text, we show that the primary states would satisfy all the geometric conditions. Like the primary state, descendant states can be viewed as descendant operators acting on the vacuum. There are infinite descendant states in a Verma module $\mathcal{V}(h, c)$. In this paper, we only focus on some special examples that are calculable, e.g., the state $\left|\psi_{1}\right\rangle:=\partial^{m} \phi(0)|0\rangle$ and $\left|\psi_{2}\right\rangle:=\partial^{m-2} \tilde{\phi}(0)|0\rangle$, where $\tilde{\phi}:=$ $(T \phi)-\frac{3}{4 h+2} \partial^{2} \phi$ is the quasiprimary operator with conformal dimension $h_{\phi}+2$.

We could calculate the one-point function $\langle T\rangle_{\partial^{m} \phi}$ and $\langle\mathcal{A}\rangle_{\partial^{m} \phi}$ by using the results in Ref. [35],

$$
\begin{aligned}
\langle T\rangle_{\partial^{m} \phi}= & \frac{\pi^{2}\left[c-24\left(m+c \epsilon_{\phi}\right)\right]}{6 L^{2}}, \\
\langle\mathcal{A}\rangle_{\partial^{m} \phi}= & \frac{\pi^{4}}{180 L^{4}\left(c \epsilon_{\phi}+1\right)\left(2 c \epsilon_{\phi}+1\right)}\left[10\left(1-24 \epsilon_{\phi}\right)^{2} \epsilon_{\phi}^{2} c^{4}+\epsilon_{\phi}\left(480\left(90 m^{2}+28 m+3\right) \epsilon_{\phi}^{2}-6(120 m+29) \epsilon_{\phi}+5\right) c^{3}\right. \\
& \left.+\left(480\left(30 m^{3}+18 m^{2}+3 m-1\right) \epsilon_{\phi}-(240 m-22)\right) c+480 m\left(6 m^{2}-1\right)\right],
\end{aligned}
$$

where we define the order $c^{0}$ constant $\epsilon_{\phi}=h_{\phi} / c$. For the states with heavy descendant that is $m=\tilde{m} c$, where $\tilde{m} \sim O\left(c^{0}\right)$, we have $h_{\phi}+m \sim c$ and

$$
\lim _{c \rightarrow \infty} \frac{\langle\mathcal{A}\rangle_{\partial^{m} \phi}-\langle T\rangle_{\partial^{m} \phi}^{2}}{c^{2}}=\frac{8 \tilde{m} \pi^{4}\left(\tilde{m}+\epsilon_{\phi}\right)\left(5 \tilde{m}+8 \epsilon_{\phi}\right)}{L^{4} \epsilon_{\phi}} \neq 0 .
$$

Even for $m \sim O\left(c^{0}\right)$, the condition (B12) is not satisfied, that is,

$$
\lim _{c \rightarrow \infty} \frac{1}{c^{2}}\left(\langle\mathcal{I}\rangle_{\partial^{m} \phi}-4\langle\mathcal{D}\rangle_{\partial^{m} \phi}\langle T\rangle_{\partial^{m} \phi}+12\langle\mathcal{A}\rangle_{\partial^{m} \phi}\langle T\rangle_{\partial^{m} \phi}^{2}\langle\mathcal{A}\rangle_{\partial^{m} \phi}^{2}-6\langle T\rangle_{\partial^{m} \phi}^{4}\right)=\frac{6144 \pi^{8} m(m+1) \epsilon_{\psi}^{2}}{L^{8}} \neq 0,
$$

for $m \neq 0$. For the state $\left|\psi_{2}\right\rangle$ with $m \sim O\left(c^{0}\right)$, we have

$$
\begin{aligned}
\lim _{c \rightarrow \infty} & \frac{1}{c^{2}}\left(\langle\mathcal{I}\rangle_{\partial^{m} \tilde{\phi}}-4\langle\mathcal{D}\rangle_{\partial^{m} \tilde{\phi}}\langle T\rangle_{\partial^{m} \tilde{\phi}}+12\langle\mathcal{A}\rangle_{\partial^{m} \tilde{\phi}}\langle T\rangle_{\partial^{m} \tilde{\phi}}^{2}\langle\mathcal{A}\rangle_{\partial^{m} \tilde{\phi}}^{2}-6\langle T\rangle_{\partial^{m} \tilde{\phi}}^{4}\right) \\
= & \frac{768 \pi^{8}\left[8\left(m^{2}-3 m+10\right) \epsilon_{\phi}{ }^{2}+16 \epsilon_{\phi}+1\right]}{L^{8}} \neq 0 .
\end{aligned}
$$

We will not give the explicit results for state $\left|\partial^{m} T\right\rangle$ and $\left|\partial^{m} \mathcal{A}\right\rangle$.

[1] J. M. Maldacena, The Large N limit of superconformal field theories and supergravity, Adv. Theor. Math. Phys. 2, 231 (1998); Int. J. Theor. Phys. 38, 1113 (1999).

[2] A. Almheiri, X. Dong, and B. Swingle, Linearity of holographic entanglement entropy, J. High Energy Phys. 02 (2017) 074.

[3] M. J. S. Beach, J. Lee, C. Rabideau, and M. Van Raamsdonk, Entanglement entropy from one-point functions in holographic states, J. High Energy Phys. 06 (2016) 085.

[4] V. Balasubramanian, B. Czech, K. Larjo, D. Marolf, and J. Simon, Quantum geometry and gravitational entropy, J. High Energy Phys. 12 (2007) 067.
[5] M. Rangamani and T. Takayanagi, Holographic entanglement entropy, Lect. Notes Phys. 931, 1 (2017).

[6] J. D. Brown and M. Henneaux, Central charges in the canonical realization of asymptotic symmetries: An example from three-dimensional gravity, Commun. Math. Phys. 104, 207 (1986).

[7] M. Banados, Three-dimensional quantum geometry and black holes, AIP Conf. Proc. 484, 147 (1999).

[8] S. Ryu and T. Takayanagi, Holographic Derivation of Entanglement Entropy from AdS/CFT, Phys. Rev. Lett. 96, 181602 (2006). 
[9] V. E. Hubeny, M. Rangamani, and T. Takayanagi, A covariant holographic entanglement entropy proposal, J. High Energy Phys. 07 (2007) 062.

[10] M. Bañados, C. Teitelboim, and J. Zanelli, The Black Hole in Three-Dimensional Space-Time, Phys. Rev. Lett. 69, 1849 (1992).

[11] X. Dong, The gravity dual of Rényi entropy, Nat. Commun. 7, 12472 (2016).

[12] B. Chen, J.-B. Wu, and J.-j. Zhang, Short interval expansion of Rényi entropy on torus, J. High Energy Phys. 08 (2016) 130.

[13] F.-L. Lin, H. Wang, and J.-j. Zhang, Thermality and excited state Rényi entropy in two-dimensional CFT, J. High Energy Phys. 11 (2016) 116.

[14] S. He, F.-L. Lin, and J.-j. Zhang, Subsystem eigenstate thermalization hypothesis for entanglement entropy in CFT, J. High Energy Phys. 08 (2017) 126.

[15] S. He, F.-L. Lin, and J.-j. Zhang, Dissimilarities of reduced density matrices and eigenstate thermalization hypothesis, J. High Energy Phys. 12 (2017) 073.

[16] P. Calabrese and J. L. Cardy, Entanglement entropy and quantum field theory, J. Stat. Mech. 06 (2004) P06002.

[17] M. Headrick, Entanglement Rényi entropies in holographic theories, Phys. Rev. D 82, 126010 (2010).

[18] P. Calabrese, J. Cardy, and E. Tonni, Entanglement entropy of two disjoint intervals in conformal field theory II, J. Stat. Mech. 01 (2011) P01021.

[19] M. Rajabpour and F. Gliozzi, Entanglement entropy of two disjoint intervals from fusion algebra of twist fields, J. Stat. Mech. 02 (2012) P02016.

[20] B. Chen and J.-j. Zhang, On short interval expansion of Rényi entropy, J. High Energy Phys. 11 (2013) 164.

[21] B. Chen, J. Long, and J.-j. Zhang, Holographic Rényi entropy for CFT with $W$ symmetry, J. High Energy Phys. 04 (2014) 041.

[22] R. Sasaki and I. Yamanaka, Virasoro algebra, vertex operators, quantum sine-Gordon and solvable quantum field theories, Adv. Stud. Pure Math. 16, 271 (1988).
[23] T. Eguchi and S.-K. Yang, Deformations of conformal field theories and Soliton equations, Phys. Lett. B 224, 373 (1989).

[24] B. A. Kupershmidt and P. Mathieu, Quantum Korteweg-de Vries like equations and perturbed conformal field theories, Phys. Lett. B 227, 245 (1989).

[25] P. Di Francesco, P. Mathieu, and D. Sénéchal, Conformal Field Theory (Springer, New York, 1997).

[26] C. T. Asplund, A. Bernamonti, F. Galli, and T. Hartman, Holographic entanglement entropy from 2d CFT: Heavy states and local quenches, J. High Energy Phys. 02 (2015) 171.

[27] P. Caputa, J. Simón, A. Štikonas, and T. Takayanagi, Quantum entanglement of localized excited states at finite temperature, J. High Energy Phys. 01 (2015) 102.

[28] A. L. Fitzpatrick, J. Kaplan, and M. T. Walters, Virasoro conformal blocks and thermality from classical background fields, J. High Energy Phys. 11 (2015) 200.

[29] E. Hijano, P. Kraus, E. Perlmutter, and R. Snively, Semiclassical Virasoro blocks from $\mathrm{AdS}_{3}$ gravity, J. High Energy Phys. 12 (2015) 077.

[30] M. Nozaki, T. Numasawa, and T. Takayanagi, Holographic local quenches and entanglement density, J. High Energy Phys. 05 (2013) 080.

[31] A. L. Fitzpatrick, J. Kaplan, M. T. Walters, and J. Wang, Hawking from Catalan, J. High Energy Phys. 05 (2016) 069.

[32] S. Goldstein, J. L. Lebowitz, R. Tumulka, and N. Zanghi, Canonical Typicality, Phys. Rev. Lett. 96, 050403 (2006).

[33] S. Popescu, A. J. Short, and A. Winter, Entanglement and the foundations of statistical mechanics, Nat. Phys. 2, 754 (2006).

[34] P. Kraus and A. Maloney, A Cardy formula for three-point coefficients or how the black hole got its spots, J. High Energy Phys. 05 (2017) 160.

[35] W.-Z. Guo, F.-L. Lin, and J. Zhang, Note on ETH of descendant states in 2D CFT, J. High Energy Phys. 01 (2019) 152. 